\title{
Dermatological and Genital Manifestations of Lyme Disease Including Morgellons Disease
}

\author{
Marianne J Middelveen (iD) \\ John S Haggblad ${ }^{2}$ \\ Julie Lewis ${ }^{3}$ \\ Gilles A Robichaud (D) ${ }^{3}$ \\ Roberto M Martinez ${ }^{4}$ \\ Jyotsna S Shah $\mathbb{D}^{5}$ \\ Iris Du Cruz $\mathbb{D}^{5}$ \\ Melissa C Fesler ${ }^{6}$ \\ Raphael B Stricker $\mathbb{D}^{6}$ \\ 'Atkins Veterinary Services, Calgary, $A B$, \\ Canada; ${ }^{2}$ Associate Clinic, Calgary, $A B$, \\ Canada; ${ }^{3}$ Department of Chemistry and \\ Biochemistry, Université de Moncton, \\ Moncton, NB, Canada; ${ }^{4}$ Martinez \\ Veterinary Services, Calgary, AB, Canada; \\ ${ }^{5}$ IGeneX Laboratories, Palo Alto, CA, \\ USA; ${ }^{6}$ Union Square Medical Associates, \\ San Francisco, CA, USA
}

Correspondence: Raphael B Stricker Union Square Medical Associates, 450 Sutter Street, Suite I504, San Francisco, CA, 94108 , USA

Tel + I 415 399- 1035

$\mathrm{Fax}+1415399-1057$

Email rstricker@usmamed.com

\begin{abstract}
Although the erythema migrans (EM) skin rash is traditionally considered a hallmark of Lyme disease, other dermatological manifestations of the tickborne disease are less well known. We describe a 49-year-old woman with erosive genital ulcerations, secondary EM rashes and jagged skin lesions associated with Lyme disease. The skin rashes exhibited fibers characteristic of Morgellons disease. Molecular testing confirmed the presence of Borrelia DNA in both vaginal culture and serum specimens. In further studies on a secondary EM lesion containing filaments, Gömöri trichrome staining revealed the presence of collagen in the filaments, while Dieterle and anti-Borrelia immunostaining revealed intracellular and extracellular Borrelia organisms. Intracellular staining for Borrelia was also observed in lymphocytic infiltrates. Lyme disease may present with a variety of genital lesions and dermatological manifestations including Morgellons disease. Careful evaluation is required to determine the presence of Borrelia organisms associated with these dermopathies.
\end{abstract}

Keywords: Lyme disease, Morgellons disease, erythema migrans, Borrelia burgdorferi, tickborne disease, spirochetes

\section{Introduction}

Tickborne Borrelia spirochetes that cause disease in animals and humans worldwide are categorized into two groups: the Lyme Disease (LD) group encompassing B. burgdorferi sensu stricto (Bbss) plus various B. burgdorferi sensu lato (Bbsl) species, and the Relapsing Fever Borrelia (RFB) group. These spirochetes are the etiological agents of Lyme disease (LD) and relapsing fever (RF), respectively. ${ }^{1-3}$

Borrelia species from both groups have been linked to an emerging, controversial, globally occurring dermopathy commonly referred to as Morgellons disease (MD). ${ }^{4-9}$ The key diagnostic criterion of this skin condition is the presence of unusual and often colorful filaments that lie under, are embedded in, or project from skin. These thread-like cutaneous filaments may be white, black, or brightly colored in hues such as red, green or blue. ${ }^{4-9}$ In addition to the curious dermopathy, MD patients may also exhibit a variety of systemic symptoms consistent with LD including fatigue, joint pain, and neurological problems. $^{4-9}$

Studies have established that $\mathrm{MD}$ is associated with $\mathrm{Bb}$ and/or RFB infection. ${ }^{4-12}$ Histological studies have revealed that MD lesions demonstrate hyperplasia and parakeratotic hyperkeratosis, and that the characteristic cutaneous fibers are composed of keratin and collagen originating primarily from keratinocytes within the stratum basale. ${ }^{13,14}$ Thus, MD fibers are of undeniable human cellular origin and are not manufactured artifacts. 
Borrelia infection is associated with a full spectrum of skin lesions and disorders including erythema migrans (EM) rash, acrodermatitis chronica atrophicans, Borrelia lymphocytoma, eosinophilic fasciitis, benign lymphocytic infiltration (Jessner-Kanof syndrome), granuloma annulare, erythema multiforme, urticarial vasculitis, infantile papular acrodermatitis (Gianotti-Crosti syndrome), morphea, lichen sclerosus et atrophicus, sclerodermatous lesions, cutaneous B-cell lymphoma, erosive genital lichenoid ulceration, and panniculitis. ${ }^{15-17}$ These skin manifestations of LD are reminiscent of secondary syphilis, which is associated with a spectrum of skin pathology including macular, papular, follicular, lichenoid, vesicular or pseudovesicular, psoriasiform, corymbiform, annular, pustular, rupial, frambesiform, pigmentary, and condylomata lata lesions. ${ }^{14}$ MD lesions can likewise demonstrate wide variation in gross morphological features that resemble those of LD-associated and syphilitic lesions. ${ }^{14}$

We describe a patient diagnosed with Borrelia infection who exhibited lesions that varied in morphology and skin distribution and also displayed fibers meeting the key diagnostic criterion of MD.

\section{Materials and Methods}

Patient informed consent for testing was obtained in accordance with the specimen collection protocol approved by the Western Institutional Review Board (WIRB), Puyallup, WA. Confidentiality of patient testing was strictly maintained. Additional written consent for publication was obtained from the patient.

Borrelia was cultured from vaginal secretions or vulvar skin scrapings by inoculation of samples in BarbourStoner-Kelly H-complete medium (BSK) with 6\% rabbit serum (Sigma Aldrich, \#B8291) and the following antibiotics: phosphomycin $(0.02 \mathrm{mg} / \mathrm{mL}$; Sigma Aldrich), rifampicin $(0.05 \mathrm{mg} / \mathrm{mL}$; Sigma Aldrich), and amphotericin B $(2.5 \mu \mathrm{g} / \mathrm{mL}$; Sigma Aldrich), as previously described. ${ }^{10,18}$ The vaginal sample was collected for culture by swabbing inside the vagina with a sterile cottontipped swab. The swab was used to inoculate BSK in a screw-capped tube filled so that it contained minimal airspace, thus providing a microaerobic environment. The culture was incubated at $32^{\circ} \mathrm{C}$, and fluid was examined by bright-field and/or dark-field microscopy for visible motile spirochetes weekly for 4 weeks. Cultures were harvested by centrifuging the fluid at $15,000 \mathrm{~g}$ for 20 minutes to concentrate spirochetal growth, retaining the pellet and discarding the supernatant.
Common Gram-positive cocci (Streptococcus and Staphylococcus spp) and Gram-negative bacteria (Escherichia coli and Klebsiella), incubated at $37^{\circ} \mathrm{C}$, under aerobic conditions, were also harvested for comparison purposes as negative controls, to exclude antigenic cross-reactivity with normal flora.

\section{IGeneX Laboratories}

Blood and skin samples were forwarded to IGeneX Laboratories, Milpitas, CA, for tickborne disease testing. Serological and PCR testing was performed as follows:

\section{IGeneX Serology}

\section{Borrelia Western Blot (WB) Testing}

The WB assays to detect IgM and IgG antibodies reactive to $\mathrm{Bb}$ and $\mathrm{RFB}$ were performed as previously described. $^{2,19}$ Nitrocellulose strips were prepared from whole-cell lysates of Bbss strains B31 and 297. Nitrocellulose strips for RFB WBs were prepared from lysates of Borrelia hermsii and Borrelia turcica that were cross-reactive with RFB species known to infect humans. Antibodies detected by this RFB WB assay detected reactivity to RFB group antigens but did not differentiate at the species level.

$\mathrm{Bb}$ and RFB strips were reacted with serum from the test subject alongside strips reacted with positive control sera from cases with confirmed LD and RFB and alongside negative control sera from uninfected subjects. Strips were soaked for 5 minutes in $1 \mathrm{~mL}$ of diluent $(100 \mathrm{mM}$ Tris, $0.9 \% \mathrm{NaCl}, 0.1 \%$ Tween $20 \%, 1 \%$ nonfat dry milk); then reacted with $10 \mu \mathrm{L}$ aliquots of test or control serum. The strips were incubated for 1 hour at ambient temperature, then washed 3 times with wash buffer (KPL, Gaithersburg, MD, USA). Strips were then incubated for 1 hour with alkaline phosphate-conjugated goat antihuman $\operatorname{IgG}$ and $\operatorname{IgM}$ (KPL), at a dilution of 1:10,000 for $\operatorname{IgG}$ and 1:6,000 for IgM, then washed thrice. To see bands, strips were reacted with 5-bromo-4-chloro-3-indolylphosphatenitro-blue tetrazolium (BCIP/NBT; KPL). Enzymatic reaction was terminated by washing with distilled water, at the point in which a calibration control produced a visible band at $39-\mathrm{kDa}$. An alkaline phosphatase-conjugated rabbit antibody to the 39/93-kDa Bbsl antigens (Strategic Biosciences, Stow, MA, USA) diluted in human serum was used as a calibration control, as previously described. ${ }^{19}$ The bands lower in intensity than that of the calibration control were reported as negative. 
$\mathrm{Bb} \mathrm{WB}$ reactivity was reported as positive if two bands from the following six antigens were present: 23-, 31-, 34-, 39, 41- and 93-kDa. Exceptions to these rules were as follows: For the IgG WB, if only the 31- and 41- or 31- and $93-\mathrm{kDa}$ bands were present then the assay was reported as indeterminate. For the IgM WB, if only the 41- and 93-kDa bands were present then the assay was interpreted as negative. Positive and negative controls were run with all $\mathrm{Bb}$ WB tests. ${ }^{19}$

WBs using separate strips for the $B$. hermsii and B. turcica species were performed for RFB WB testing to determine IgM and IgG reactivity. IgM or IgG was interpreted as positive if two bands from the following four antigens were reactive: 21-23-, Glycerophosphodiester phosphodiesterase (GlpQ), 41- and 70-75-kDa. Positive and negative controls were run with all RFB WB assays. ${ }^{2}$

\section{Tickborne Coinfection Testing}

Serological testing for tickborne coinfections including Babesia, Ehrlichia and Bartonella was performed using standard immunofluorescence assay (IFA) methods, as described by the laboratory (https://igenex.com/knowledge-center/).

\section{IGeneX PCR of Blood Samples}

Multiplex PCR was performed to detect $\mathrm{Bb}$ in blood and serum, as previously described. ${ }^{11,20}$ Quantitative PCR (qPCR) targeting a 16S rDNA fragment was performed for the detection of RFB species in clinical specimens, as previously described. ${ }^{2,12}$

\section{Université de Moncton PCR of Vaginal Culture Samples}

Vaginal culture samples were forwarded to the Department of Chemistry and Biochemistry, Université de Moncton, Moncton, NB. DNA extracted from the samples was analyzed using qPCR with FlaB and OspA primers, as previously described. ${ }^{21}$ Samples were run in triplicate together with a positive control spiked with different $\mathrm{Bb}$ concentrations into $100 \mathrm{ng}$ of HEK cell line DNA and a negative control (no template), as previously described. ${ }^{21}$

\section{McClains Laboratories}

\section{Histopathology Testing of Biopsy Samples}

A biopsy exhibiting embedded and protruding fibers taken from the margin of an expanding lesion resembling a secondary EM rash was formalin-fixed and forwarded to McClains Laboratories LLC, Smithtown, NY, where it was paraffin-blocked, sectioned and stained. Biopsy sections were stained with hematoxylin and eosin (H\&E), Gömöri trichrome,
Dieterle silver nitrate and Gram stains using standard laboratory protocols. Sections were also stained with anti-Bb immunostain (Abcam, Cambridge, MA), as previously described. ${ }^{10,18} \mathrm{Bb}$ immunostaining, as reported by the manufacturer, cross-reacts to other Borrelia spp. and Treponema spp. Control staining, as previously described, demonstrated no reactivity to common Gram-positive and Gram-negative organisms, nor was it reactive to fungal-infected human skin specimens or inflamed skin specimens from a patient diagnosed with psoriasis. ${ }^{14}$

\section{Case Report}

A 49-year-old Indo-Aryan female presented at a Calgary clinic in early March 2015 complaining of vaginal itching of several weeks duration. At the time of presentation she had been living part time in the province of Alberta and part time in the province of Manitoba. Examination revealed an external vaginal and vulvar papular red rash, and an area surrounding the vagina displayed skin loss, hypopigmentation, cracks, fissures, and ulceration. Similar hypopigmented patches of skin occurred on her breasts. She was treated with oral fluconazole for 3 days while awaiting swab and culture results. Multiple vaginal/cervical swabs were obtained and the differential diagnosis included Trichomonas infection, yeast infection, bacterial vaginosis, Chlamydia infection, gonorrhea, varicella-zoster virus and herpes simplex virus (HSV). Testing was positive for HSV type 1. Based on these results she was treated with valacyclovir. By late March 2015 her symptoms had improved but were unresolved with genital fissures and ulceration still present. Test results from March 2015 were positive for IgG antibodies to HSV type 1 or 2 . The possibility of lichen planus (LP) was explored but a genital biopsy was not performed.

In June 2015, she presented with unresolved gynecological symptoms and, in addition, had developed ulcerated, punctate erosions with ragged edges on her back (Figure 1). Microscopic examination of the back lesions at 50X magnification revealed unusual black fibers embedded in skin tissue, meeting the key diagnostic criterion for MD. Due to the association between MD and LD, the possibility of LD was explored. Although she did not recall a tick bite or characteristic EM rash, she recalled having an unidentified arthropod bite on her right forearm in the summer of 2014, and she admitted to having taken frequent walks through a large local wooded park in Alberta at the time when the bite occurred. She recalled a bite that was red, itchy, deeper than a typical mosquito bite, and that took longer than a month to disappear.

Serum, whole blood, and skin specimens were forwarded to IGeneX Laboratories for serological and PCR testing in 


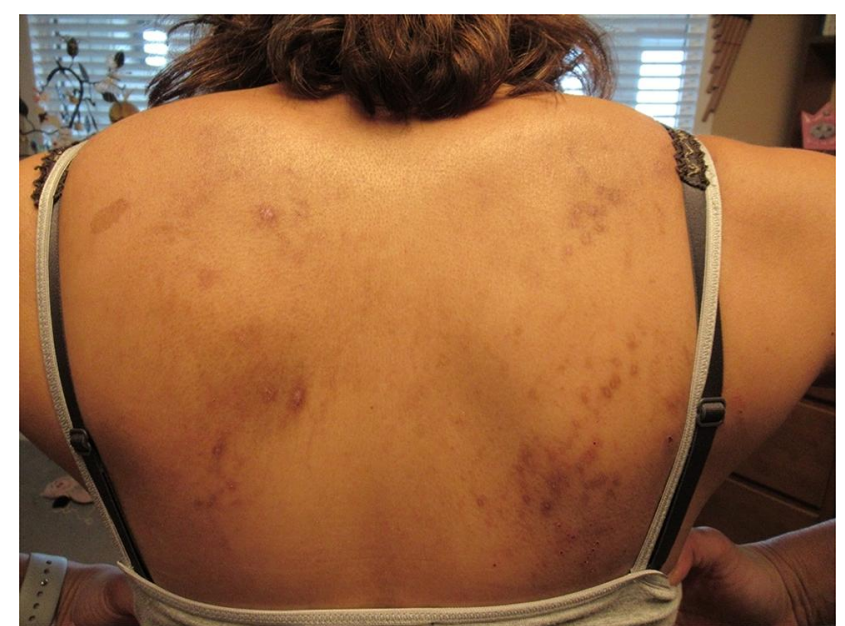

Figure I Ulcerated, punctate erosions with ragged edges on the patient's back typical of Morgellons disease lesions.

June 2015. Antibodies to both Bbsl and RFB species and $\mathrm{Bb}$ DNA were detected in the serum sample. Vaginal Borrelia culture samples were forwarded to Université de Moncton for Bb PCR testing, and the samples yielded positive results for the presence of Bbsl genes when run with appropriate positive and negative controls, as described in Materials and Methods.

In August 2016, the patient presented with pustular open sores on her shoulders and arms. As her symptoms had progressed she was treated with doxycycline 100mg BID for 3 months. Treatment with doxycycline resulted in clinical improvement but not resolution, so treatment was extended to 6 months. In January 2017, antibiotic treatment was discontinued because it had failed to completely resolve her symptoms. Serological tests were positive for Bb, Babesia duncani and Ehrlichia chaffeensis (Table 1). Based on her clinical symptoms and test results she was treated with azithromycin 500mg QD and nitazoxanide 500mg BID. By July 2017 her symptoms were much improved, and her lesions had mostly resolved, although a few minor lesions containing fibers were still present. Everyday function improved and she maintained full-time employment as an accountant and cared for her two children. She reported flare-ups of skin lesions if she consumed sugar or processed foods or became stressed.

In October 2017, she had an outbreak of acne-like lesions on her chin consistent with perioral dermatitis. Azithromycin and nitazoxanide treatment was discontinued and treatment with doxycycline was resumed. By December 2017, her skin condition and other symptoms had worsened, so doxycycline treatment was switched back to azithromycin and nitazoxanide. Repeat serological testing performed in December 2017 was negative for Ehrlichia chaffeensis but positive for $\mathrm{Bb}$, Babesia duncani, and Bartonella henselae (Table 1). Treatment with azithromycin and nitazoxanide was continued as she had previously done well on this regimen.

Table I Results of Laboratory Testing

\begin{tabular}{|c|c|c|c|c|}
\hline Laboratory & Pathogen & Technique & Result & Date Performed \\
\hline Calgary Laboratory Services & HSV & Serology & + & March 2015 \\
\hline \multirow[t]{3}{*}{ IGeneX } & $\mathrm{Bb}$ & Western Blot & + & \multirow[t]{3}{*}{ June 2015} \\
\hline & RFB & Western Blot & + & \\
\hline & $\mathrm{Bb}$ & PCR Skin Specimen & + & \\
\hline Université de Moncton & $\mathrm{Bb}$ & PCR Vaginal Culture & + & $\begin{array}{l}\text { Cultured June } 2015 \\
\text { PCR performed September } 2020\end{array}$ \\
\hline \multirow[t]{6}{*}{ IGeneX } & $\mathrm{Bb}$ & Western Blot & + & \multirow[t]{3}{*}{ January 2017} \\
\hline & B. duncani & IFA & + & \\
\hline & E. chaffeensis & IFA & + & \\
\hline & $\mathrm{Bb}$ & Western Blot & + & \multirow[t]{3}{*}{ December 2017} \\
\hline & B. duncani & IFA & + & \\
\hline & B. henselae & IFA & + & \\
\hline McClains Laboratories & $\mathrm{Bb}$ & Immunostains Biopsy & + & December 2018 \\
\hline
\end{tabular}

Notes: Details of tickborne disease testing are available at https://igenex.com/knowledge-center/.

Abbreviations: HSV, herpes simplex virus; Bb, Borrelia burgdorferi; RFB, relapsing fever Borrelia; IFA, immunofluorescence assay. 
In December 2018, antibiotic treatment was terminated. The patient then relapsed with the development of annular rashes on her hands, legs, back and abdomen (Figure 2A-C).

\section{A}

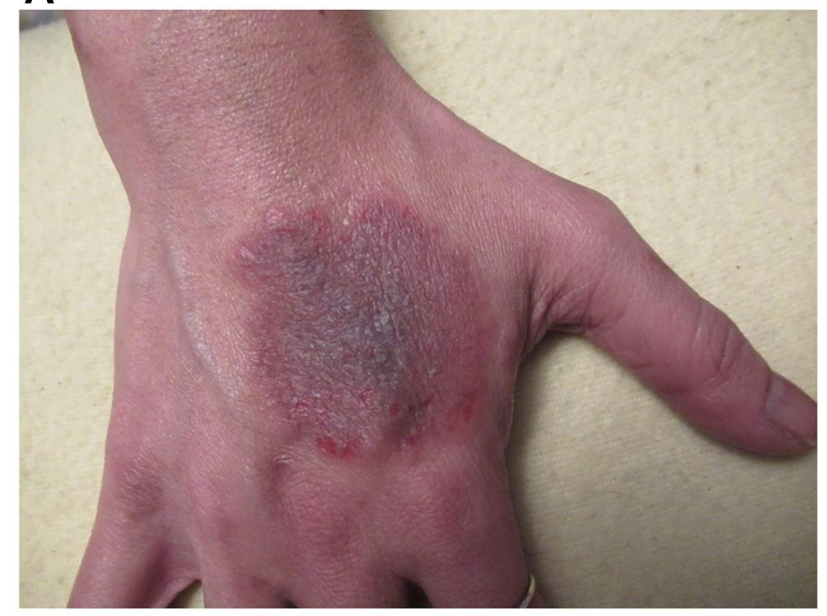

B

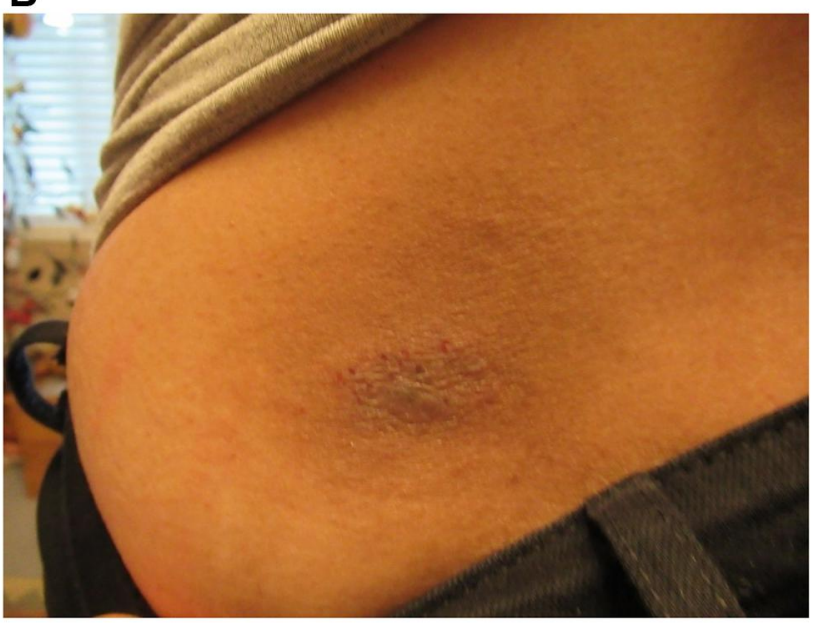

C

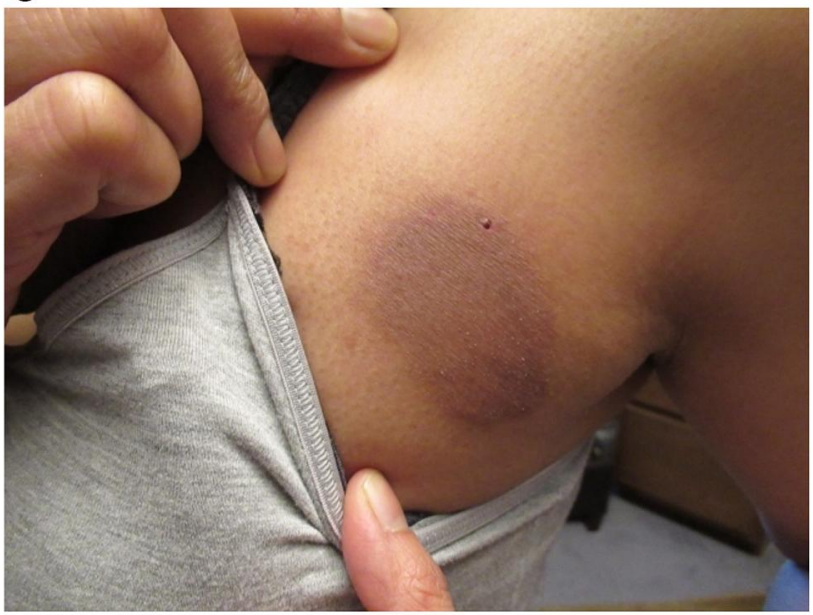

Figure 2 (A-C) Expanding annular rashes on the patient's hands, back and leg, with a raised, advancing, erythematous border consistent with secondary erythema migrans (EM) rashes.
These lesions started at less than $1 \mathrm{~cm}$ and increased up to $6-7 \mathrm{~cm}$ in diameter, and the advancing border was raised and erythematous consistent with secondary EM rashes. Microscopic examination of the rashes at $100 \mathrm{X}$ magnification revealed blue embedded fibers consistent with MD (Figure 3). A biopsy taken from the advancing edge was formalin-fixed and forwarded to McClains Laboratories for histological staining (Figure 4). Sections stained with hematoxylin and eosin (H\&E) exhibited parakeratotic hyperkeratosis, spongiosis, and hemorrhage. The stratum spinosum and stratum basale demonstrated disarray and some degeneration with the formation of vacuoles and apoptotic bodies (Figure 5). Perivascular lymphocytic infiltrate was present in the

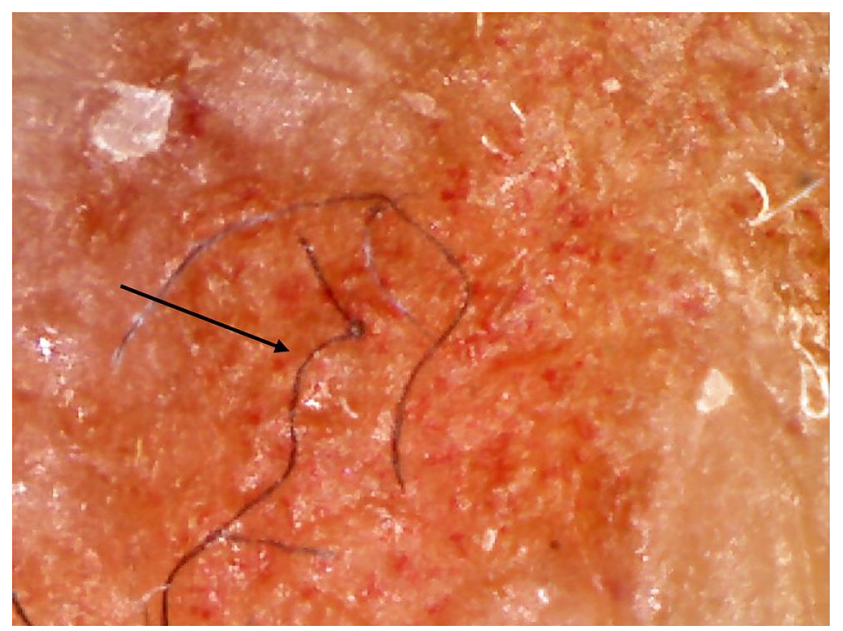

Figure 3 Secondary EM rash revealing embedded fibers (arrow) consistent with Morgellons disease. I00X original magnification.

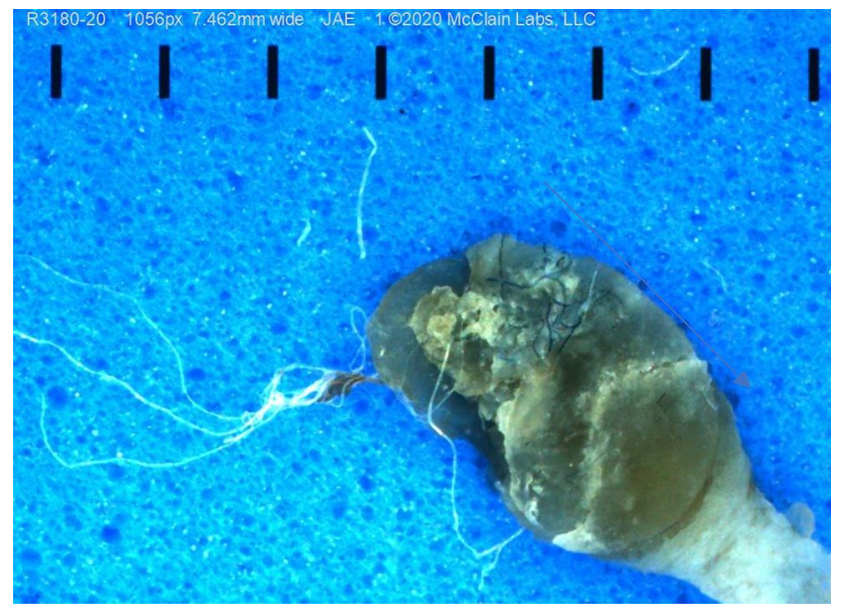

Figure 4 Gross morphology of a biopsy taken from the advancing edge of a lesion on the hand, revealing embedded and protruding blue and white fibers. I00X original magnification. 
stratum basale and dermis, indicating chronicity. Gömöri trichrome staining revealed that the fibers contained collagen. Dieterle staining revealed bacterial aggregates within the hyperkeratotic stratum corneum with both long extracellular spirochetes and intracellular organisms among the keratinocytes in the stratum basale (Figure 6A). Lymphocytes and glandular tissue within the dermis also displayed positive Dieterle intracellular staining. Anti-Bb immunostaining was positive with strongly staining bacterial aggregates in the hyperkeratotic stratum corneum. These aggregates displayed both positive and negative staining, indicating that the aggregates were composed of a mixture of different bacterial species (Figure 6B).

Aggregates generally displayed a central positively stained core surrounded by a sheath of negatively stained bacteria. Positively stained Borrelia organisms consistent with cystic morphology were visible within many of the bacterial aggregates. Positive intracellular and extracellular staining was prominent within the keratinocytes of the stratum basale and stratum spinosum (Figure 7A). The lymphocytes in dermal infiltrates and cells within dermal glandular tissue revealed positive anti- $\mathrm{Bb}$ intracellular staining (Figure 7B and $\mathrm{C}$ ). Anti$\mathrm{Bb}$ staining of fungal-infected skin served as a negative control (Figure 7D). Gram staining revealed Grampositive cocci scattered on the superficial stratum corneum and in bacterial aggregates within the hyperkeratotic stratum corneum (Figure 8A). The deeper layers of the epidermis and dermis were devoid of Gram-positive organisms (Figure 8B). These findings indicate Borrelia organisms were present in mixed-bacterial aggregates,

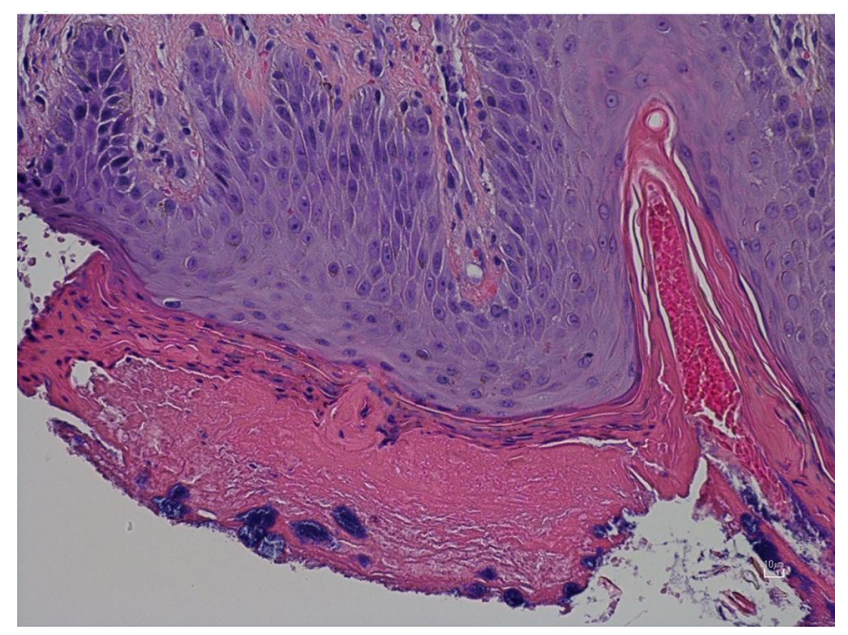

Figure 5 Sections stained with H\&E exhibiting parakeratotic hyperkeratosis, spongiosis, and hemorrhage. 200X original magnification.
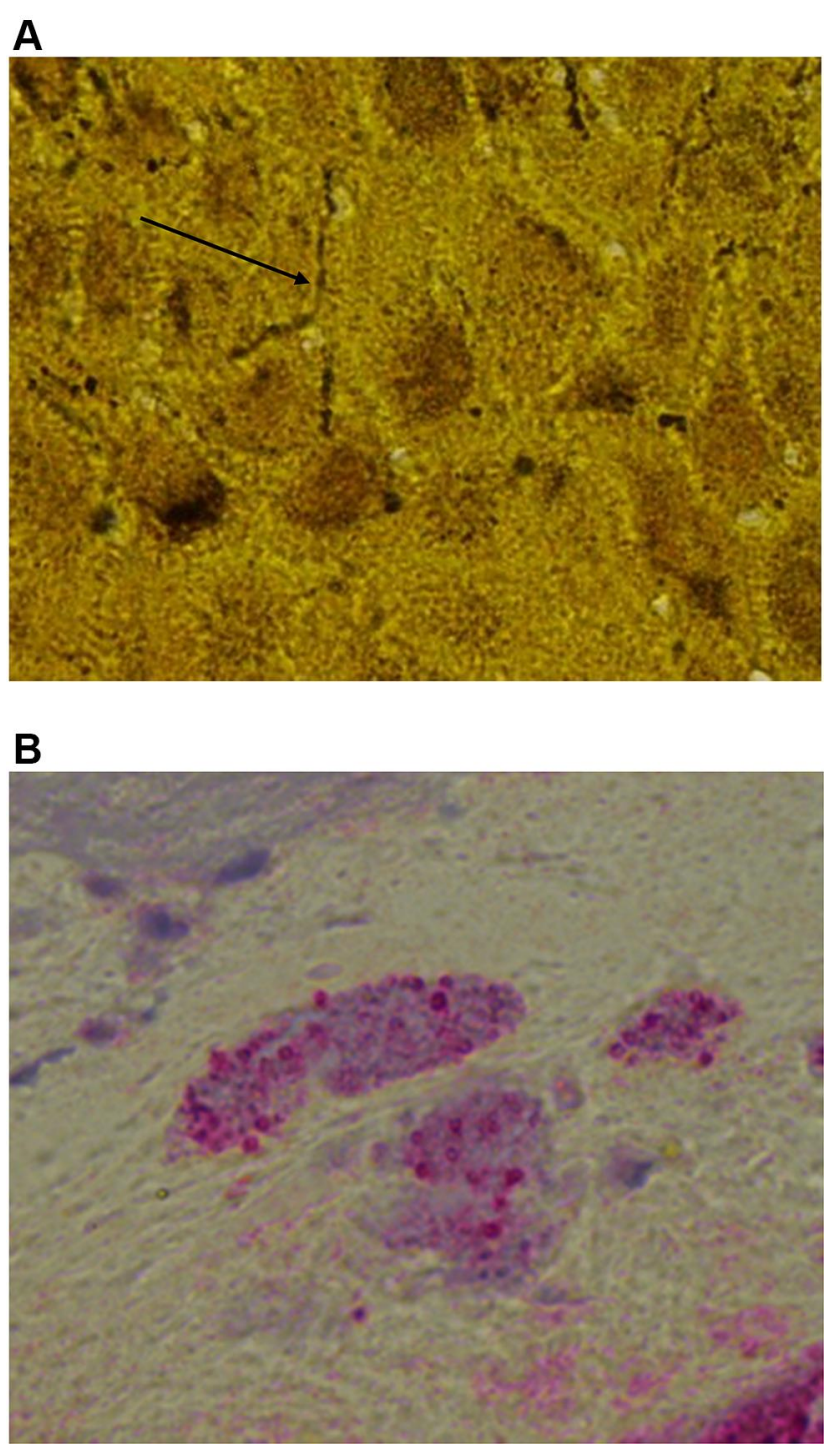

Figure 6 (A) Dieterle silver stain of spirochetes (arrow) among basal keratinocytes. 1000X original magnification. (B) Anti-Bb immunostained section showing positive-stained Borrelia organisms consistent with cystic morphology mixed with negatively stained bacteria within bacterial aggregates. $1000 \mathrm{X}$ original magnification.

and both intra- and extracellularly among the keratinocytes of the stratum basale, consistent with the different morphological variants of $\mathrm{Bb}$ described previously. ${ }^{10,22-28}$ Gram-positive cocci were limited to the upper layers of the epidermis only.

Based on clinical observation and test results, treatment with azithromycin and nitazoxanide was resumed along with a short course of prednisone. By June 2018 the patient reported feeling very well, but she reported that her skin would still break out if she consumed sugar or processed foods. Her current treatment consists of a cycling maintenance regimen of tinidazole $500 \mathrm{mg}$ BID and trimethoprim/sulfamethoxazole DS BID. She 


\section{A}
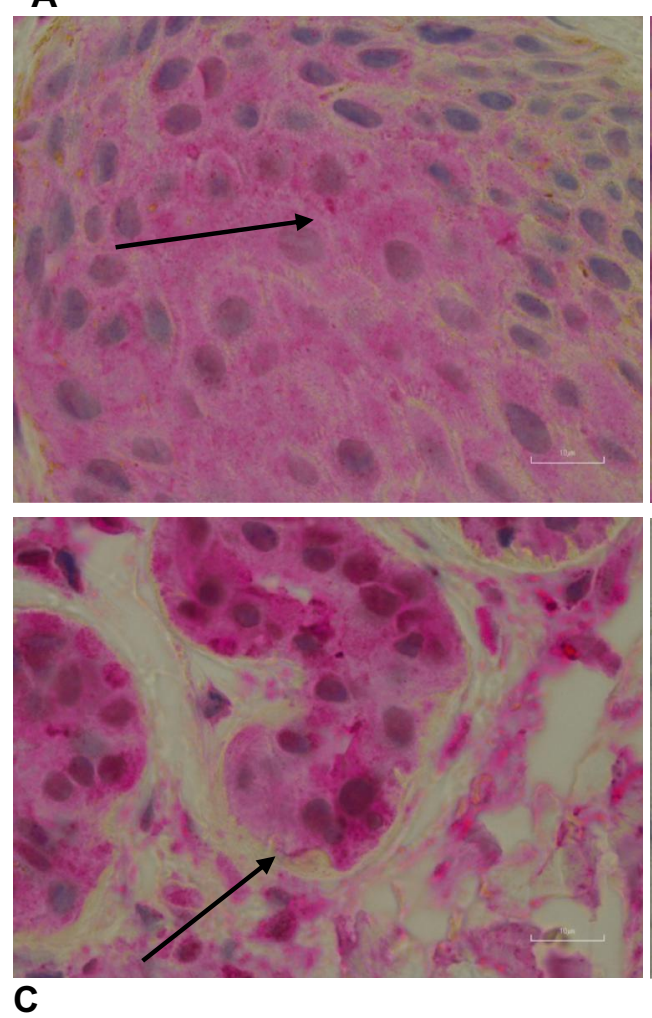

B
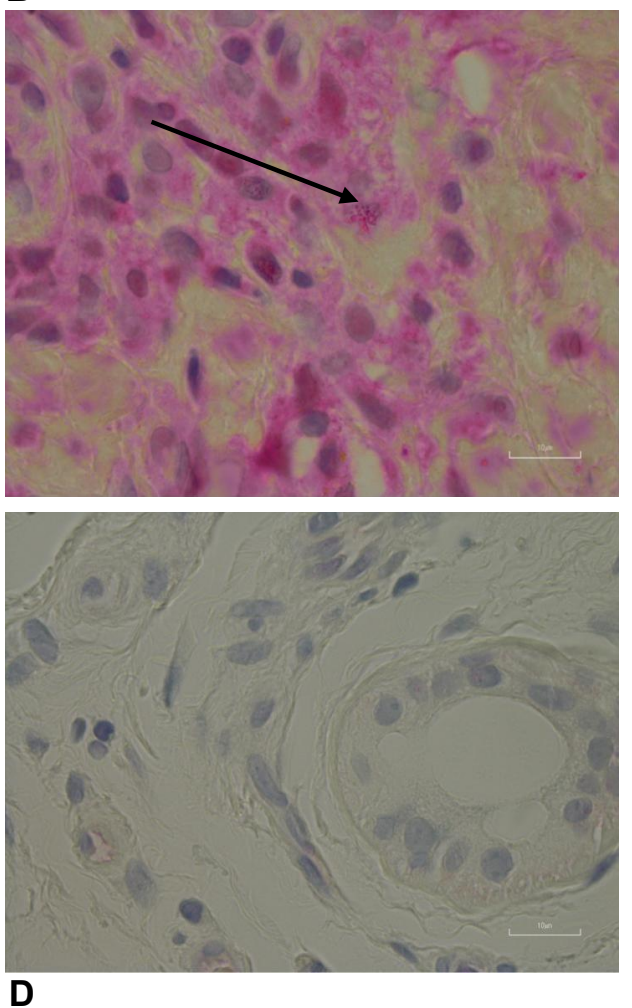

Figure 7 (A) Anti-Bb immunostained section showing positively stained intracellular and extracellular organisms (arrow) associated with keratinocytes of the stratum basale and stratum spinosum. 1000X original magnification. (B) Anti-Bb immunostained section showing intracellular staining of organisms within lymphocytes (arrow) in a dermal infiltrate. 1000X original magnification. (C) Anti-Bb immunostained section showing positively stained organisms (arrow) in dermal glandular tissue. I000X original magnification. (D) Anti-Bb immunostained control section of fungal-infected skin. 1000X original magnification.

reports her status at present as being well and functional.

\section{Discussion}

In medicine, disease staging is used to provide objective medical criteria to categorize and assess disease severity and progression. ${ }^{14}$ Syphilis, a well-known spirochetal infection, is categorized in three sequential stages accompanied by an array of corresponding lesions. ${ }^{14,29,30}$ The primary stage of syphilis is characterized by genital chancres. Secondary syphilis is associated with an assortment of lesions, in terms of gross morphological features, that reflect progressive stages of infection. Tertiary syphilis is associated with neurological deterioration. ${ }^{14,29,30}$

Borrelia spirochetal infections are also linked to a wide variety of lesions that, like syphilitic lesions, reflect progressive stages of infection. Dermatological conditions linked to LD include primary and secondary EM rash, Borrelia lymphocytoma, acrodermatitis chronica atrophicans, morphea, lichen planus, lichen sclerosus, cutaneous
B-cell lymphoma, scleroderma, lymphadenosis cutis, and prurigo pigmentosa. ${ }^{10,12,14,16,31-33}$ If categorized in a manner comparable to the staging of syphilis, the primary stage of LD is characterized by an EM rash, an expanding lesion that occurs approximately 3-30 days after Borrelia infection via a tick bite. ${ }^{15}$ The EM rash is thought to occur in only a fraction of LD cases. If untreated, roughly $20-25 \%$ of LD patients go on to present with multiple rashes caused by hematogenous spread of the infection. The secondary stage of LD is said to occur between 3-8 months and can be characterized by the variety of skin disorders outlined above. ${ }^{15}$ Tertiary LD develops after a minimum of 7 months of infection, and a variety of skin conditions are characteristic of this stage, including acrodermatitis chronica atrophicans, lichen sclerosus et atrophicus, morphea, atrophoderma, and eosinophilic fasciitis. ${ }^{15}$

Temporal and morphological variation like that of syphilis has been observed in MD lesions. ${ }^{4,5,10,14}$ The following clinical classification system was proposed, based on the duration and location of MD lesions: 


\section{A}

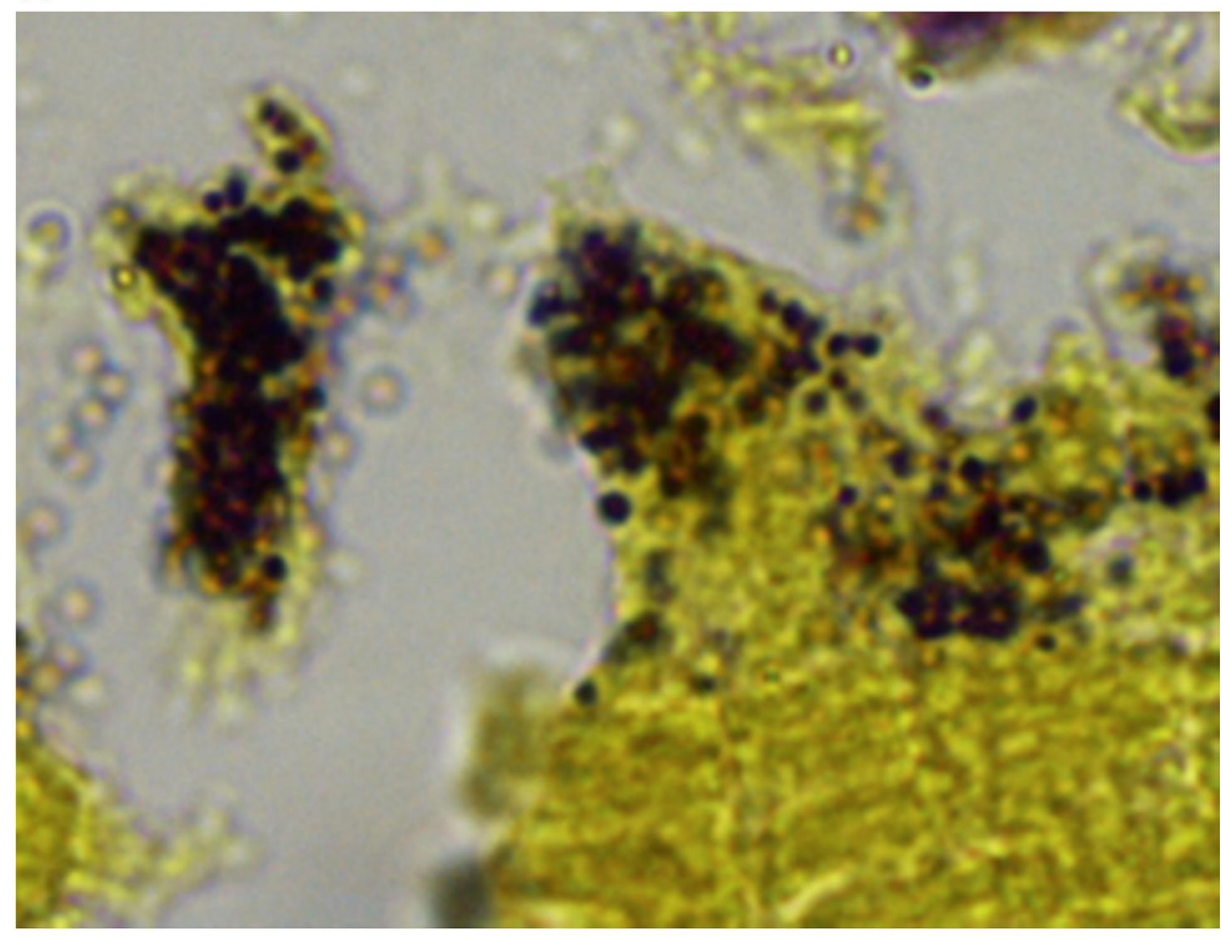

B

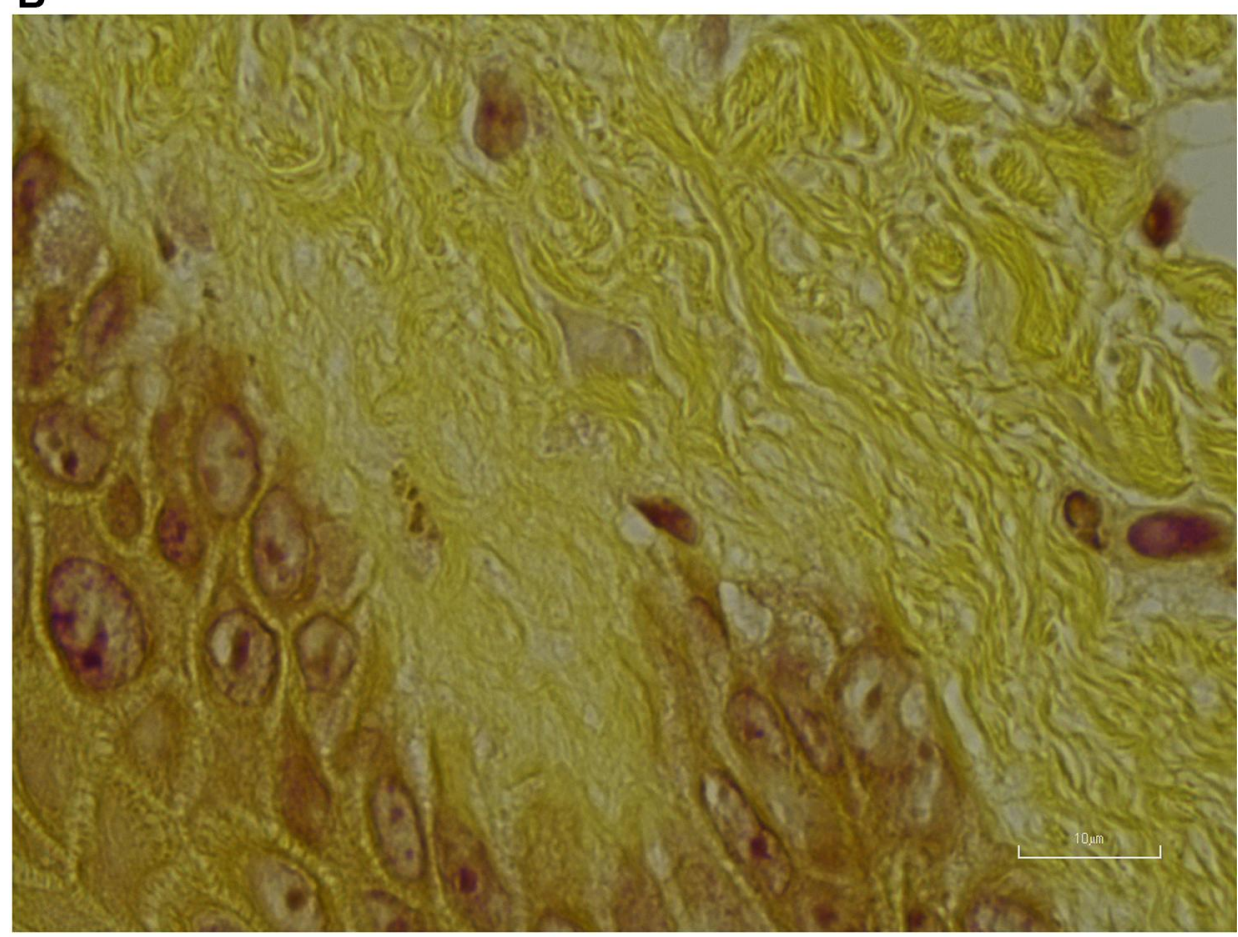

Figure 8 (A) Gram stain of biopsy section showing Gram-positive cocci in superficial layer. 1000X original magnification. (B) Gram stain of biopsy section showing negative staining in deep layers. $1000 \mathrm{X}$ original magnification.

Class I (Early localized): lesions/fibers present for less than three (3) months and localized to one area of the body (head, trunk, extremities).
Class II (Early disseminated): lesions/fibers present for less than three (3) months and involving more than one area of the body (head, trunk, extremities). 
Class III (Late localized): lesions/fibers present for more than six (6) months and localized to one area of the body (head, trunk, extremities).

Class IV (Late disseminated): lesions/fibers present for more than six (6) months and involving more than one area of the body (head, trunk, extremities).

Further staging of clinical classes was proposed based on histopathology and anti-Bb immunostaining:

Stage A: Lesions demonstrate little or no immune infiltrates and little or no disorganization of cells; macrophages are not present, and hemorrhage is negligible. Extracellular isolated spirochetes and intracellular staining of keratinocytes in the lower epidermis are occasionally seen.

Stage B: Lesions demonstrate a pattern intermediate between Stages A and C.

Stage C: Lesions demonstrate positive staining of keratinocytes in the stratum basale and stratum spinosum and positive intracellular staining of macrophages for Borrelia. Aggregate Borrelia colonies are frequently encountered, hemorrhage is frequent, and intracellularly stained fibroblasts are occasionally seen.

The patient in this study was followed through progressive stages of infection with a variety of associated lesions (Figures 1-5). At present she would be classified clinically based on the extent of disease and histopathologically based on her secondary EM lesions as Class IV (Late Disseminated) Stage C. ${ }^{14}$

The patient recalled a slow-healing arthropod bite in 2014, a year before developing any further symptoms. This arthropod bite may have been the point of initial infection equivalent to the primary stage of LD infection. She did not develop any further symptoms until the following year when she presented with vulvovaginitis. She was not examined for the presence of fibers associated with the vulvovaginitis; therefore, it is not known if this dermatological problem was consistent with MD. However, a vaginal culture resulted in the growth of spirochetes identified as Bb by PCR analysis. Borrelia has been implicated in the development of vulvovaginitis and genital ulceration. ${ }^{17,34}$ Thus, the gynecological problems that the subject experienced were likely related to $\mathrm{Bb}$ infection.

Histological studies were not performed on the lesions demonstrating cutaneous filaments that occurred on her back. We did, however, study sections of a biopsy taken from one of the expanding secondary EM rashes (Figures 6 and 7). These secondary EM rashes exhibited embedded and projecting fibers typical of MD lesions. ${ }^{13}$ Histology of the secondary EM rash differed from the Stage C MD lesions previously studied and categorized in that there was a lymphocytic infiltrate that demonstrated intracellular staining, and spongiosis was present. The histology did resemble Stage $\mathrm{C}$ in that hemorrhage, positive staining of keratinocytes in the stratum basale and stratum spinosum, and aggregate Borrelia colonies were present.

The histology observed in our case study is consistent with published descriptions of EM rashes. Biopsies from the center of these rashes show slight papillary edema with superficial and deep perivascular lymphohistiocytic infiltrate. ${ }^{35}$ In addition, a lymphocytic infiltrate at the dermoepidermal junction with disruption of the basement membrane may be present. ${ }^{35}$ Biopsies from the erythematous advancing border of EM rashes can demonstrate a perivascular lymphohistiocytic infiltrate, occasionally mixed with plasma cells. ${ }^{35}$ Biopsies at the periphery of an expanding EM lesion can demonstrate histopathological variation, including spongiosis, the presence of neutrophils and eosinophils, focal interface change, involvement of the superficial plexus alone, and the absence of plasma cells. ${ }^{15}$

When stained by methods that reveal spirochetes, EM rashes typically do not present high numbers of $\mathrm{Bb}$ spirochetes as would be seen in lesions associated with Treponema pallidum infection, and the $\mathrm{Bb}$ microorganisms are present in different morphological forms such as cystic structures. ${ }^{35}$ Dieterle staining and $\mathrm{Bb}$ immunostaining were performed on the biopsy of a secondary EM rash in this study, revealing the presence of occasional extracellular Borrelia spirochetes as well as other extracellular and intracellular Borrelia variants including organisms with cystic morphology that express Borrelia antigens. In later stages of infection, $\mathrm{Bb}$ organisms may be found in as few as half of EM and lymphocytoma biopsies studied. ${ }^{35}$ In contrast, the biopsy from our patient frequently revealed spirochetes and other morphological forms of Borrelia. Borrelia are consistently found and identified in MD lesions. ${ }^{10,12}$ Perhaps secondary factors leading to the evolution of MD contribute to the establishment of high spirochetal loads. Gram staining revealed that cocci were present only on the surface of the stratum corneum and within mixed bacteria/Borrelia aggregates. The different bacteria present in mixed bacterial aggregates can behave in a synergistic manner, thus enhancing biofilm production. ${ }^{12}$ The fact that aggregates in this study had a central Borrelia core surrounded by Gram positive cocci 
and perhaps other organisms suggests that organisms present in the aggregate occupied different biological niches.

This patient had positive testing for HSV-type 1 and the tickborne coinfections Babesia duncani and Ehrlichia chaffeensis. In addition, mixed bacterial aggregates were observed in the epidermis of the EM rash biopsy. These infections may have added to the complexity of this patient's illness and may have been contributing factors in the development and evolution of the various rashes displayed throughout the illness. We speculate that spirochetal infection by Borrelia species is the key etiologic factor in MD evolution, as Borrelia are the pathogens most consistently detected in MD skin specimens. ${ }^{5,6,10,11}$ However, MD patients frequently test positive for other co-infecting tickborne diseases. ${ }^{4-7,9,11,14}$ Furthermore, the co-infecting tickborne pathogens Bartonella henselae and Rickettsia spp. and the common human pathogens Helicobacter pylori and Treponema denticola have been detected directly in MD skin specimens. ${ }^{5,6,10,11,14}$ The role of each pathogen detected in clinical MD specimens and its contribution to the disease process remains to be determined, but these bacteria likely contribute to the establishment of the disease and its progression, severity and persistence. ${ }^{5,6,10,11,14}$

This patient had rashes exhibiting morphological variation and fiber formation consistent with MD. The patient had no prior knowledge of $\mathrm{MD}$, and she was unaware of the presence of cutaneous fibers in her lesions. The presence of fibers in lesions matching the clinical definition of MD was first noted by her primary care physician upon physical examination with 50X magnification of skin lesions, and on that basis the diagnosis of MD was made. Her primary care physician knew of the association between MD and LD, and that led him to test for and diagnose LD, RF and other tickborne diseases. Had the patient not developed filamentous cutaneous lesions, she would most likely have continued undiagnosed and untreated.

This patient had positive testing for spirochetes from both the LD and RFB groups of Borrelia. In Canada and in the USA, the exact number of people becoming infected by Borrelia spirochetes is unknown. In the USA, approximately 35,000 cases of Lyme disease are reported each year, yet the US Centers for Disease Control and Prevention (CDC) states that as many as 476,000 people are diagnosed with $\mathrm{LD}$ each year. ${ }^{36} \mathrm{~A}$ recent Canadian study based on tick surveillance and canine infection rates in the province of New Brunswick postulated a 12-58 fold underestimation of human LD cases when applied across the country (only $1.7 \%$ to $8.3 \%$ of cases detected). ${ }^{37}$ The prevalence of RFB cases is also uncertain as there is no standard case definition for relapsing fever, and it is not nationally reportable in the USA. ${ }^{38}$ In North America, most cases are reported in the western USA, southern British Columbia and northern Mexico. $B$. hermsii and $B$. turicatae are the RFB species most commonly reported. ${ }^{3,39,40}$

LD-associated lesions may be mistaken for other unrelated dermatological conditions. Many LD patients may unknowingly have filamentous lesions meeting the key diagnostic criterion for MD. Unless 50X or higher magnification is used to examine lesions, cutaneous filaments may go unnoticed by patients and physicians. This study underscores the importance of looking closely at dermatological lesions at 50X or higher magnification. If unusual cutaneous fibers are present, it is imperative to consider the possibility of LD and other tickborne diseases.

Optimal antibiotic therapy has yet to be determined for MD. Despite long courses of antibiotics, the patient remained symptomatic and developed recurrence of symptoms when antibiotics were discontinued. Borrelia is known to evade the immune system and can remain in privileged sites despite treatment. Cutaneous infection may allow Borrelia to escape host defenses and antibiotic treatment. Further studies are required to understand this complex dermopathy and to evaluate the best treatment options for LD and RFB in patients who present with MD.

\section{Conclusions}

Lyme disease may present with a variety of genital and dermatological manifestations. Careful evaluation is required to determine the presence of Borrelia organisms associated with these diverse lesions. If skin lesions contain fibers, the diagnosis of MD should be considered and testing for tickborne disease should be undertaken. Relapsing Fever Borrelia and tickborne coinfections are being recognized more frequently in association with dermatological manifestations, and it is important to consider these emerging infections to ensure optimal treatment and patient improvement.

\section{Acknowledgments}

Supported by a grant from the Called2Serve Foundation, Provo, UT. This article is dedicated to the memory of Lucy Middelveen-Allan. 


\section{Disclosure}

JSS is the President and stock owner of IGeneX Inc. RBS is the owner of Union Square Medical Associates. MJM and RBS are owners of US Patent No.10,613,084 pertaining to Lyme disease. The authors report no other conflicts of interest in this work.

\section{References}

1. Todar K. Todar's Online Textbook of Bacteriology. Madison, WI, USA: University of Wisconsin-Madison, Department of Bacteriology; 2006.

2. Middelveen MJ, Shah JS, Fesler MC, Stricker RB. Relapsing fever Borrelia in California: a pilot serological study. Int J Gen Med. 2018;11:373-382. doi:10.2147/IJGM.S176493

3. Fesler MC, Shah JS, Middelveen MJ, Du Cruz I, Burrascano JJ, Stricker RB. Lyme disease: diversity of Borrelia species in California and Mexico detected using a novel immunoblot assay. Healthcare (Basel). 2020;8(2):97. doi:10.3390/healthcare8020097

4. Savely VR, Stricker RB. Morgellons disease: analysis of a population with clinically confirmed microscopic subcutaneous fibers of unknown etiology. Clin Cosmet Investig Dermatol. 2010;3:67-78. doi:10.2147/ccid.s9520

5. Middelveen MJ, Stricker RB. Morgellons disease: a filamentous borrelial dermatitis. Int J Gen Med. 2016;9:349-354. doi:10.2147/IJGM. S116608

6. Middelveen MJ, Fesler MC, Stricker RB. History of Morgellons disease: from delusion to definition. Clin Cosmet Investig Dermatol. 2018;11:71-90. doi:10.2147/CCID.S152343

7. Middelveen MJ, Stricker RB. Filament formation associated with spirochetal infection: a comparative approach to Morgellons disease. Clin Cosmet Investig Dermatol. 2011;4:167-177. doi:10.2147/CCID.S26183

8. Middelveen MJ, Mayne PJ, Kahn DG, Stricker RB. Characterization and evolution of dermal filaments from patients with Morgellons disease. Clin Cosmet Investig Dermatol. 2013;6:1-21. doi:10.2147/CCID.S39017

9. Fesler MC, Middelveen MJ, Stricker RB. Clinical evaluation of Morgellons disease in a cohort of North American patients. Dermatol Rep. 2018;10(1):7660. doi:10.4081/dr.2018.7660

10. Middelveen MJ, Bandoski C, Burke J, et al. Exploring the association between Morgellons disease and Lyme disease: identification of Borrelia burgdorferi in Morgellons disease patients. BMC Dermatol. 2015;15:1. doi:10.1186/s12895-015-0023-0

11. Middelveen MJ, Cruz ID, Fesler MC, Stricker RB, Shah JS. Detection of tick-borne infection in Morgellons disease patients by serological and molecular techniques. Clin Cosmet Investig Dermatol. 2018;11:561-569. doi:10.2147/CCID.S184521

12. Middelveen MJ, Burke J, Sapi E, et al. Culture and identification of Borrelia spirochetes in human vaginal and seminal secretions. F1000Res. 2014;3:309. doi:10.12688/f1000research.5778.3

13. Middelveen MJ, Rasmussen EH, Kahn DG, Stricker RB. Morgellons disease: a chemical and light microscopic study. J Clin Exp Dermatol Res. 2012;3:140. doi:10.4172/2155-9554.1000140

14. Middelveen MJ, Martinez RM, Fesler MC, et al. Classification and staging of Morgellons disease: lessons from syphilis. Clin Cosmet Investig Dermatol. 2020;13:145-164. doi:10.2147/CCID.S239840

15. Wilson TC, Legler A, Madison KC, Fairley JA, Swick BL. Erythema migrans: a spectrum of histopathologic changes. Am J Dermatopathol. 2012;34:834-837. doi:10.1097/DAD.0b013e31825879be

16. Vasudevan B, Chatterjee M. Lyme borreliosis and skin. Indian J Dermatol. 2013;58:167-174. doi:10.4103/0019-5154.110822
17. Fesler MC, Middelveen MJ, Burke JM, Stricker RB. Erosive vulvovaginitis associated with Borrelia burgdorferi infection. J Investig Med High Impact Case Rep. 2019;7:2324709619842901. doi:10.1177/2324709619842901

18. Middelveen MJ, McClain SA, Bandoski C, et al. Granulomatous hepatitis associated with chronic Borrelia burgdorferi infection: a case report. Research Open Access. 2014;1:875. doi:10.13070/rs.en.1.875

19. Shah JS, Cruz DI, Narcisco W, Lo W, Harris NS. Improved sensitivity of Lyme disease Western blots prepared with a mixture of Borrelia burgdorferi strains 297 and B31. Chronic Dis Int. 2014;1(2):7.

20. Shah JS, D'Cruz I, Ward S, Harris NS, Ramasamy R. Development of a sensitive PCR-dot blot assay to supplement serological tests for diagnosing Lyme disease. Eur J Clin Microbiol Infect Dis. 2018;37 (4):701-709. doi:10.1007/s10096-017-3162-x

21. Wills MKB, Kirby AM, Lloyd VK. Detecting the Lyme disease spirochete, Borrelia burgdorferi, in ticks using nested PCR. $J$ Vis Exp. 2018;132:56471. doi:10.3791/56471

22. Kurtti TJ, Munderloh UG, Johnson RC, et al. Colony formation and morphology in Borrelia burgdorferi. J Clin Microbiol. 1987;25 (11):2054-2058. doi:10.1128/JCM.25.11.2054-2058.1987

23. Mursic VP, Wanner G, Reinhardt $S$, et al. Sphäroplasten-L PhaseVarianten von Borrelia burgdorferi: morphologische Analyse und Kultivierung. Infection. 1996;24(3):218-226. doi:10.1007/BF01781096

24. Brorson $\varnothing$, Brorson S, Scythes J, et al. Destruction of spirochete Borrelia burgdorferi round-body propagules (RBs) by the antibiotic tigecycline. Proc Natl Acad Sci U S A. 2009;106(44):18656-18661. doi:10.1073/pnas.0908236106

25. Barthold SW, Hodzic E, Imai DM, et al. Ineffectiveness of tigecycline against persistent Borrelia burgdorferi. Antimicrob Agents Chemother. 2010;54(2):643-651. doi:10.1128/AAC.00788-09

26. MacDonald AB. Borrelia burgdorferi tissue morphologies and imaging methodologies. Eur J Clin Microbiol Infect Dis. 2013;32 (8):1077-1082. doi:10.1007/s10096-013-1853-5

27. Hodzic E, Imai D, Feng S, et al. Resurgence of persisting non-cultivable Borrelia burgdorferi following antibiotic treatment in mice. PLoS One. 2014;9(1):e86907. doi:10.1371/journal.pone.0086907

28. Meriläinen L, Herranen A, Schwarzbach A, et al. Morphological and biochemical features of Borrelia burgdorferi pleomorphic forms. Microbiology. 2015;pii:mic.0.000027. doi:10.1099/mic.0.000027

29. Baughn RE, Musher DM. Secondary syphilitic lesions. Clin Microbiol Rev. 2005;18(1):205-216. doi:10.1128/CMR.18.1.205-216.2005

30. French P. Syphilis. BMJ. 2007;334:143-147. doi:10.1136/ bmj.39085.518148.BE

31. Afa G, Caprilli F, Crescimbeni E, Morrone A, Progano G, Fazio M. Anti-Borrelia burgdorferi antibodies in chronic erythema migrans, benign lymphadenosis cutis, scleroderma and scleroatrophic lichen. G Ital Dermatol Venereol. 1990;125:369-373.

32. Malane MS, Grant-Kels JM, Feder HM, Luger SW. Diagnosis of Lyme disease based on dermatologic manifestations. Ann Intern Med. 1991;114:490-498. doi:10.7326/0003-4819-114-6-490

33. Chao LL, Lu CF, Shih CM. Molecular detection and genetic identification of Borrelia garini and Borrelia afzelii from patients presenting a rare skin manifestation of prurigo pigmentosa in Taiwan. Int J Infect Dis. 2013;17:1141-1147. doi:10.1016/j.ijid.2013.08.004

34. Finch JJ, Wald J, Ferenczi K, Khalid S, Murphy M. Disseminated Lyme disease presenting with nonsexual acute genital ulcers. JAMA Dermatol. 2014;150(11):1202-1204. doi:10.1001/jamadermatol.2014.1072

35. De Koning J. Histopathologic patterns of erythema migrans and borrelial lymphocytoma. Clin Dermatol. 1993;11(3):377-383. doi:10.1016/0738-081x(93)90093-r

36. Centers for Disease Control and Prevention. How many people get Lyme disease? 2021. Available from: https://www.cdc.gov/lyme/ stats/humancases.html. Accessed April 14, 2021.

37. Lloyd VK, Hawkins RG. Under-detection of Lyme disease in Canada. Healthcare (Basel). 2018;6(4):125. doi:10.3390/healthcare6040125 
38. Forrester JD, Kjemtrup AM, Fritz CL. Tickborne relapsing fever-United States, 1990-2011. MMWR. 2015;64:58-60.

39. Dworkin MS, Schwan TG, Anderson DE. Tick-borne relapsing fever in North America. Med Clin North Am. 2002;86(2):417-ix. doi:10.1016/s0025-7125(03)00095-6
40. Colunga-Salas P, Sánchez-Montes S, Volkow P, Ruíz-Remigio A, Becker I. Lyme disease and relapsing fever in Mexico: an overview of human and wildlife infections. PLoS One. 2020;15(9):e0238496. doi:10.1371/journal.pone. 0238496

\section{Publish your work in this journal}

Clinical, Cosmetic and Investigational Dermatology is an international, peer-reviewed, open access, online journal that focuses on the latest clinical and experimental research in all aspects of skin disease and cosmetic interventions. This journal is indexed on CAS.

Submit your manuscript here: https://www.dovepress.com/clinical-cosmetic-and-investigational-dermatology-journal
The manuscript management system is completely online and includes a very quick and fair peer-review system, which is all easy to use. Visit http://www.dovepress.com/testimonials.php to read real quotes from published authors. 